indwelling catheter obviates the loss of echogenicity due to "unstable contrast." Also, saline microbubbles may wash out earlier than perfluorobutane microbubbles, thus creating less interference with further imaging. We thank Drs. Urade and Fukumoto for referring to their recent papers, in which they described "pseudostaining" of the liver parenchyma caused by perfluorobutane microbubbles leading to accurate identification of the duct. In our study, use of intrabiliary saline sharply demarcated the segment(s) to which the duct belongs and was clearly visible as parenchymal echogenicity on ultrasound imaging. Primarily, this is how we have identified the duct in our technique and is very similar to "pseudostaining." The echogenicity caused by saline may be less intense than perflourobutane microbubbles; however, it still sharply demarcates the respective segment(s). Necessity is the mother of invention, and we propose that our technique will be of value to a hepatobiliary surgeon when making intraoperative decisions with minimal resources.

Abhijit Chandra, MD, MCh
Affiliation: From the Department of Surgical Gastroenterology, King George Medical University, Lucknow, India.

DOI: $10.1503 /$ cjs. 1861012

\section{Correction of PLACE of PRACTICE: BRAMPTON, NOT BRANTFORD}

I read with interest the editorial in the December 2017 issue, "Dr. Louis Kristal at 100: witness to the evolution of surgery in Canada." I practised orthopedic surgery in Brampton from 1974 to 2010. I knew Lou well as a GP/anesthesiologist and subsequently as a GP surgical assistant. I enjoyed his discussions with interested physicians in the "Doctor's Lounge" on Saturday mornings. He was a staunch Liberal, and I was always careful to steer clear of some of the political controversies at the time.

I am pleased to see he has an excellent memory at 100 !

Dr. Kristal's practice in 1974 for 10 years was in Brampton, not Brantford, as the article states.

Donald Prior, MD
Affiliations: Former Chief of Surgery (retired), Peel Memorial Hospital and William Osler Health Centre, Brampton, Ont.

Competing interests: None declared.

DOI: $10.1503 /$ cjs.1861013

\section{References}

1. V. McAlister. Dr. Louis Kristal at 100: witness to the evolution of surgery in Canada. Can 7 Surg 2017;60:364-5.

\section{AUTHOR RESPONSE}

Many thanks to Dr. Prior for his letter about our recent editorial on Dr. Louis Kristal. The journal has corrected the place of practice in the online version of the article.

Regrettably, Dr. Kristal passed away, not long after the editorial was published, on Dec. 8, 2017.

\section{Vivian McAlister, MB}

Affiliation: Coeditor, Canadian fournal of Surgery.

DOI: $10.1503 /$ cjs.1861014 